\title{
Post-Multikulturalismus und „repressive Autonomie“: sozialanthropologische Perspektiven zur Integrationsdebatte ${ }^{1}$
}

\author{
Sabine Strasser, Universität Bern
}

Nach turbulenten medialen und politischen Debatten über das „Scheitern des Multikulturalismus“" wurden seit der Jahrtausendwende in ganz Europa neue Maßnahmen zur Integration von zugewanderten Minderheiten eingeleitet. Für diese Neuorientierungen und im Zuge einer steten Vervielfältigung von Herkunfts- und Zielregionen von MigrantInnen und von Rechtstiteln und sozialen Positionen von Zugewanderten wurde in den letzten Jahren der Begriff Post-Multikulturalismus (King 2005; Uitermark et al. 2005; Vertovec 2010) geprägt. Damit verbunden waren neue Forderungen nach sozialer Kohäsion, gemeinsamen Vorstellungen von Demokratie und geteilten Werten, ohne dabei den respektvollen Umgang mit Diversität zu verdrängen und mit dem Ziel sozio-ökonomische Mobilität zu fördern. Dieser Begriff verbindet die gegenwärtigen Forderungen nach Integration in Ländern wie Großbritannien und den Niederlanden mit ihrer multikulturalistischen Vergangenheit und lenkt dort den Blick auf mögliche Kontinuitäten und notwendige Neuorientierungen. In Ländern wie Österreich, Deutschland oder der Schweiz verweist der Begriff allerdings vor allem auf den Diskurs des Anti-Multikulturalismus, der den Multikulturalismus selbst dort für räumliche Segregation, Arbeitslosigkeit oder hohe Drop-out-Raten in Schulen verantwortlich gemacht hat, wo dieser nie explizit rechtliche Norm oder dominanter Diskurs gewesen ist. In der Folge des „Abschieds vom Multikulturalismus“ wurden also auch in den ehemaligen „Gastarbeiterregimen“ Integrationsdebatten verstärkt, Integrationsgipfel und Dialogforen abgehalten und Indikatoren für Barometer und Monitoren entwickelt (Hess et al. 2009; Schönwälder 2010; D’Amato 2010; Perchinig 2010; Strasser und Tošiç in diesem Band). Zeitgleich mit diesen Bemühungen, Integrationsleistungen der staatlichen und städtischen Institutionen zu messen und zu verbessern, nahm jedoch auch der Leistungsdruck auf zugewanderte Minderheiten und deren nachfolgende Generationen zu. Aus dem Fördern wurde zunehmend ein Fordern von Leistungen wie die Kenntnisse der lokalen Sprachen, der Landeskunde, der Geschichte; der politischen Systeme und der Grundwerte.

\footnotetext{
${ }^{1}$ Ich bedanke mich bei Jelena Tošić für ihre anregende Kritik und Durchsicht des Manuskripts und bei Elisabeth Holzleithner, Christa Markom und Ines Rössl für die Erarbeitung wesentlicher Grundlagen von dem Zusammenhang Post-Multikulturalismus und repressiver Autonomie im NODE-Projekt „Contesting Multiculturalism. Gender Equality, Cultural Diversity and Sexual Autonomy in the European Union, Austrian Federal Ministry of Education, Science and Culture“" (2006-2008).
} 
Die Wissenschaft begann schon vor der Jahrtausendwende, die Potentiale und Gefahren des Multikulturalismus kontrovers zu diskutieren. Während manche den Niedergang des Multikulturalismus prognostizierten (Joppke 2004), betonten andere eine eher nur rhetorische Abkehr durch die Politik, die kaum Auswirkungen auf die politischen Praktiken haben würde (Bauböck 2002; Vertovec und Wessendorf 2010). Neben den Debatten zwischen liberalen (Kymlicka 1995, 2010) und kommunitaristischen (Taylor 1993) VertreterInnen des Multikulturalismus ${ }^{2}$ um die unterschiedlichen Verflechtungen von universellen Rechten, Gleichheit und Freiheit einerseits und von kultureller Differenzen und Gruppenrechten andererseits waren in der letzten Dekade insbesondere feministische Standpunkte heftig umstritten. Während liberale Feministinnen kritisierten, dass die Politik der Zugeständnisse die bestehenden patriarchalen Machtverhältnisse im Inneren der ethnischen Minderheiten stärken würde, statt staatlichen Schutz für verletzbare oder marginalisierte Individuen zu bieten, meinten postkoloniale TheoretikerInnen, dass der liberale Diskurs selbst Frauen wie auch Lesben und Schwule innerhalb der abgewerteten Minderheiten auf jeden Fall zusätzlich schwächt, indem er sie für handlungsunfähige Opfer erklärt und damit abwertet.

Der nach dem vielfach postulierten Scheitern der multikulturalistischen Politik entstandene Begriff Post-Multikulturalismus, der von manchen AutorInnen auch als „,neue Assimilation“ (Brubaker 2001; Rattansi 2004) oder „Hochkonjunktur des Integrationsbegriffs“ (Hess et al. 2009) beschrieben wurde, soll in diesem Beitrag in seiner Entstehungsgeschichte und in seinen Effekten beleuchtet werden. Können die Maßnahmen der Politik nach dem ausgerufenen Ende des Multikulturalismus das oft genannte Ziel erreichen, im Inneren der Europäischen Union bzw. des Schengen Raumes Inklusion und Chancengleichheit zu verbessern? Werden dadurch differenziertere Antworten auf die Herausforderungen durch eine zunehmende Diversität der Bevölkerung umsetzbar? Oder werden durch diese neuen Maßnahmen nur, wie einige befürchten, die Regulierung von Zuwanderung und die Kontrolle von Zugewanderten verstärkt (Hess et al. 2009; Phillips 2007)?

Ich werde in diesem Beitrag zuerst kurz skizzieren, was unter Multikulturalismus in den Ländern verstanden wurde, wo er als Werkzeug der Politik Anwendung fand. Im Anschluß daran werde ich zeigen, welche Positionen sich in der wissenschaftlichen Debatte um den

\footnotetext{
${ }^{2}$ Nach Steven Vertovec's (2010, S.85) unterscheidet Stuart Hall (2001 zit. nach Vertovec 2010) zwischen „conservative multiculturalism“, der Differenz an die Vorstellungen von Mehrheiten anpasst, „liberal multiculturalism“, der Differenz universellen Rechten unterordnet, ,pluralist multiculturalism“ der Differenz in segmentierte soziale Ordnungen einsperrt, „,commercial multiculturalism”, der Differenzen konsumiert und deshalb das “exotische Andere als Spektakel konstruiert und schliesslich „,corporate multiculturalism” der Differenz im Interesse der dominanten Gruppe reguliert.
} 
(Anti-)Multikulturalismus verbergen und welche Bedrohungen, Warnungen und Werte die wissenschaftlichen Streitparteien gegeneinander vorbringen. Beispiele von sozial- und kulturwissenschaftlichen Positionen zum Rätsel Multikulturalismus ermöglichen, in Anlehnung an Terence Turner (1993), noch einmal die Frage aufzuwerfen, ob die Sozialanthropologie etwas zu bieten hat, dessen sich die Politik im Umgang mit der aktuellen Diversität der Bevölkerung bewußt sein sollte. Schließlich soll der Weg in den PostMultikulturalismus mit seinen Maßnahmen nachgezeichnet werden, um die Frage der Auswirkungen dieser veränderten Politik und insbesondere sein emanzipatorisches bzw. repressives Potential in Bezug auf das Geschlechterverhältnis auszuloten. Ich werde zeigen, wie Argumente des Feminismus in der post-multikulturalistischen Ära weniger der egalitären Inklusion von minorisierten Frauen, als der Abwertung, Ungleichbehandlung und Ausgrenzung von kulturell konstruierten und ethnisch segregierten Gruppen dienen. In Anlehnung an den Begriff ,,repressiver Liberalismus“ des Soziologen und Politikwissenschafters Christian Joppke (2007), der vor unerwünschten Ergebnissen bei der Durchsetzung von liberalen Forderungen mit illiberalen Mitteln warnt, verwende ich den Ausdruck „repressive Autonomie“, um die mißbräuchliche Verwendung von feministischen Argumenten für Geschlechteregalität, Selbstbestimmung und sexuelle Freiheit aufzuzeigen. Werden diese Forderungen nämlich mit illiberalen Maßnahmen verknüpft, führen diese statt zu mehr Autonomie von minorisierten Frauen zu deren Einschränkungen und Unterordnungen. Daraus lässt sich ableiten, dass der Multikulturalismus wohl eher mit Fragen sozialer Mobilität, politischer Partizipation, Anti-Diskriminierung und dynamischen Konzepten von Kultur verknüpft und komplexer gedacht statt abgelöst werden sollte. Somit wende ich mich abschließend erneut dem Multikulturalismus zu und frage, unter welchen Bedingungen multikulturalistische Politik der Komplexität gesellschaftlicher Vielfalt gerecht wird, kulturelle Anerkennung mit Forderungen nach Gleichheit und sozio-ökonomischer Mobilität verbindet und schliesslich feministische Forderungen nach Bekämpfung von Gewalt gegen Frauen ohne Abwertung von ethnischen und religiösen Minderheiten ermöglicht.

\section{Wege in den Anti-Multikulturalismus}

Der Multikulturalismus war nie ein monolithischer Block oder gar ein inhaltlich eindeutig abgegrenztes Politikfeld. Auch die Einwanderungsländer mit einer explizit multikulturalistischen Politik USA, Kanada und Australien hatten unterschiedliche 
Beweggründe, diesen politischen Ansatz zu verfolgen: Die USA reagierte mit Multikulturalismus auf die Bürgerrechtsbewegung, Kanada musste einen Weg finden, mit einer drohenden Abspaltung von Quebec umzugehen und Australien versuchte dadurch den tief verwurzelten Rassismus gegen Aborigines zu bekämpfen. In den nordamerikanischen Ländern wurde der Multikulturalismus in den 1990er Jahren schließlich als allgegenwärtiges und unausweichliches Gesellschaftsmodell bezeichnet.

In den meisten europäischen Ländern ist ethnische, nationale und religiöse Vielfalt nicht erst durch Arbeitsmigration nach dem Zweiten Weltkrieg entstanden, sondern hatte aufgrund von Kolonialgeschichte, Nationenbildungen und globalen Flüchtlingsbewegungen eine lange Vorgeschichte. Der politische Umgang mit der gesellschaftlichen Tatsache „Vielfalt“ wurde auch in einigen europäischen Ländern (wie UK, Schweden und den Niederlanden) als Multikulturalismus bezeichnet. In den meisten Ländern West- und Nordeuropas führten die Herausforderungen durch Zuwanderung und Vervielfältigung der Gesellschaft in der Praxis zu unterschiedlichen Kombinationen aus Assimilation, Segregation und wechselseitiger Anerkennung, die je nach politischem Verständnis in verschiedenen Nationalstaaten gesetzlich unterschiedlich umgesetzt wurden. Neue Herausforderungen bewirkten daher immer wieder unterschiedliche, oft hilflose Reaktionen, die sich aus heutiger Sicht als ein kontinuierliches trial and error-Verfahren darstellen. ${ }^{3}$

In der Politik gibt es ein reiches Spektrum an Aktivitäten, die unter dem Label „multikulturalistisch“ gehandelt werden: die Förderung von Kultur-, Sport- und Moscheenvereinen ethnischer Vereinigungen und religiöser Gemeinschaften, die Ausnahmeregelungen für religiöse Minderheiten wie jene der rituellen Schlachtungen, Zugeständnissen bezüglich des Speiseplans in öffentlichen Einrichtungen, Medien, in denen Stereotypen vermieden und die Möglichkeit für eigene Sendungen und Sender in unterschiedlichen Sprachen gegeben wird. Wesentlich bei diesen Aktivitäten ist die kulturelle Sensibilität in Institutionen. In der Schule zeugen Sprachförderung, angepasste Curricula oder Muttersprachenunterricht davon, bei der Polizei Lockerung der Bekleidungsvorschriften oder die Anwerbung von Mitgliedern minorisierter Gruppen bei der Rekrutierung von Beamten. Insbesondere bei Gericht sollten kultursensible Beurteilungen von Sachverhalten einen hohen Stellenwert haben. ${ }^{4}$ Öffentliche Einrichtungen wie Krankenhäuser sind angehalten Schutz vor

\footnotetext{
${ }^{3}$ Rainer Bauböck merkt beispielsweise zur Integrationspolitik in Österreich an, dass es: „(...) kaum ein[en] Ansatz der staatlichen Regelung [gibt], der nicht erprobt worden wäre“ (1997, 1).

4 „Kultur im Gerichtssaal“, also die Notwendigkeit unterschiedliche Normen zu berücksichtigen bzw. die Gefahren, die eine Anwendung von unterschiedlichen Rechtsgrundlagen nach kulturellen Vorstellungen
} 
Diskriminierung und Anreize für Gleichbehandlung zu entwickeln. Doch nicht nur kulturelle Sensibilität wird in der multikulturalistischen Politik verhandelt, sondern vor allem die Möglichkeiten die staatlichen Institutionen und öffentlichen Einrichtungen unabhängig von ethnischer Herkunft, religiöser Zugehörigkeit oder sexueller Orientierung nutzen zu können. Zudem bilden soziale Gleichheit, gerechte Umverteilungen und politische Mitbestimmung einen wichtigen Bestandteil von multikulturalistischer Anerkennungspolitik. Die Möglichkeit der Doppelstaatsbürgerschaften ist deshalb genauso ein Merkmal multikulturalistischer Politik wie die Verleihung der Staatsbürgerschaft an im Inland geborene Kinder von NichtStaatsbürgerInnen (ius solis) oder das Recht zu wählen und transnational zu heiraten. Der politische Multikulturalismus verlangt also einen umfassenden Ansatz von Gerechtigkeit in den Institutionen, der aber je nach politischer Herausforderung und nationalen Traditionen immer wieder höchst unterschiedlich Gestalt annimmt.

Kurz nachdem Norman Glazer (1997) und Will Kymlicka (1998) den Multikulturalismus als eindeutig notwendige und allen anderen überlegene Beschreibung, Analyse und Politik in komplexen Gesellschaften verkündet hatten, kam es zu einem Einbruch in der Karriere des Konzepts. Christian Joppke stellte schon kurze Zeit später sogar den Rückzug vom Multikulturalismus in Politik und Wissenschaft fest (Joppke 2004). Insbesondere nach den Anschlägen auf New York und Washington (2001) begann eine beschleunigte Debatte um Gefahren dieser Politik und die Vermischungen und Verwechslungen von Sicherheits- und Migrationsagenden. Schließlich trugen Ängste vor kulturellen Bedrohungen, räumlicher Segregation und steigende Arbeitslosigkeit immer wieder zu Forderungen nach dem Rückzug vom Multikulturalismus bei. Die multikulturalistische Politik würde demnach kulturelle Differenzen verstärken und essentialisieren, soziale Grenzziehungen vertiefen und individuelle Autonomie untergraben. In der Sprache der populistischen Politik wurden für diese identifizierten „Missstände“ je nach Kontext und Ideologie Begriffe wie Parallelgesellschaft, islamischer Terrorismus und „home-grown terror“ oder kulturelle Bedrohungen verwendet und die so markierten Gruppen zusätzlich mit Eigenschaften wie anti-demokratisch, integrationsunwillig und gewaltbereit verknüpft.

Bemerkenswert ist, dass auch in Deutschland, Österreich und der Schweiz der Multikulturalismus für „,soziale Missstände“ verantwortlich gemacht wurde, obwohl diese Politik für die Regulierung von Vielfalt keinesfalls dominant war. Auch wenn in Frankfurt ein 
„Amt für multikulturelle Angelegenheiten“ eingerichtet wurde und Institutionen in der Schweiz und in Österreich viele der oben genannten Maßnahmen auf lokaler Ebene und vor allem in den großen Städten partiell umgesetzt haben, verwiesen europäische Vergleiche von Migrationsregimen diese Länder lange Zeit in Bezug auf kulturelle Integration und politische Partizipation in ein assimilatorisch-exkludierendes Segment (Koopmans und Statham 2000). ${ }^{5}$ Der schwierige Zugang zu politischen Rechten, Institutionen und sozialen Gütern sowie immer wiederkehrende Werte- und Leitkulturdebatten bildeten zusammen mit den enormen Hürden am Weg zu einer Bürgerschaft eine Verbindung zwischen den deutschsprachigen Ländern. ${ }^{6}$

Die heftigste Kritik am Multikulturalismus kam vom Rechtspopulismus, der in vielen Ländern im Zuge der Globalisierung und gesellschaftlichen Verunsicherung durch neoliberale Wirtschaftskonzepte einen neuen Aufschwung erlebte. Begriffe wie „NeoNationalismus“ (Gingrich und Banks 2006, S.2) oder „,neuer Realismus“ (Prins und Saharso 2010, S.76ff) versuchen, die Begründungen für die neue Verteilung von Rechten und Pflichten nach deren Vorstellungen von Integration einzufangen. Andre Gingrich und Markus Banks (2006) sehen in der Neuauflage nationalistischer Bewegungen und Parteien in ganz Europa eine Reaktion auf Globalisierung und transnationale Beziehungen und untersuchen alte „,invention of traditions“ in einem neuen Kleid. Baukje Prins und Sawitri Sahraso diskutieren den Triumphzug eines Populismus, der die Enthüllung der angeblichen Lügen des Multikulturalismus auf seine Fahnen geheftet hat. Der „,neue Realismus“ stützt sich dabei auf drei Elemente: 1) die Pflicht offen zu sagen, was die gesellschaftlichen Probleme sind und dabei auch Tabus zu brechen, 2) das vollmundig zum Ausdruck gebrachte Verständnis für die einfachen Leute, insbesondere den kleinen Mann und 3) die Affirmation nationaler (westlichdemokratischer und geschlechter-egalitärer) Identität in Abgrenzung zur Bedrohung durch Islamisierung und Immigration.

\footnotetext{
${ }^{5}$ Koopmans und Statham $(2000,19)$ schlugen vier idealtypische Regime vor: 1) ethnische Segregation, 2) ethnische Assimilation, 3) republikanischer Monismus und 4) Pluralismus oder Multikulturalismus, die auf zwei Achsen Zuordnungen sowohl in Bezug auf soziale Inklusion und Exklusion als auch in Bezug auf kulturelle Assimilation und Vielfalt ermöglichen.

${ }^{6}$ Alle drei Staaten gehen vom Prinzip des ius sanguinis aus, wonach die Abstammung und nicht der Aufenthalt über die Bürgerschaft bestimmen. Der Erwerb der Staatsbürgerschaft erfolgt nach bestimmten Integrationskriterien, zu denen ein Mindesteinkommen, adäquate Wohnverhältnisse, Sprachkenntnisse und Kenntnisse von Landeskunde, Geschichte und Kultur. Sichtlich ist Einbürgerung vom Prinzip her nicht erwünscht und wird als letzter Schritt einer gelungenen Integration gesehen nicht als Beginn. Deutschland hat sich mit der Zuerkennung der deutschen Staatsbürgerschaft für im Inland geborene Kinder von Zugewanderten, die zum Zeitpunkt der Geburt länger als acht Jahre in Deutschland leben und ein unbefristetes Aufenthaltsrecht haben, am meisten von diesem Abstammungsdenken wegbewegt (Schönwälder 2010).
} 
Die Positionen der österreichischen Nationalisten Jörg Haider und H.C. Strache, des holländischen Politikers, Soziologen und (unter Hinweis auf seine eigene Homosexualität) Multikulturalismusgegners Pim Fortuyn, seiner populären somalisch-holländischen Kollegin und Islamkritikerin Ayaan Hirsi Ali bis hin zur viel beachteten türkisch-deutschen Soziologin Necla Kelek lassen alle Komponenten des neuen Realismus erkennen: Beherzt werden multikulturalistische Lügen aufgedeckt, Tabus gebrochen, der kleine Mann und die kleine Frau zum Applaus aufgefordert, wenn angebliche Integrationsverweigerer und Sozialschmarotzer angeprangert werden und der Islam und die MuslimInnen genauso wie die angeblichen Traditionen der Zugewanderten hemmungslos verurteilt. ${ }^{7}$ Doch für die Politiken der Kontrolle und des Messens, des stärker Forderns und weniger Förderns haben diese Positionen nur den Weg geebnet, die Umsetzungen eines neuen Abschnitts der Integrationspolitik wurden von Parteien der Mitte vorgenommen. Die konservativen und christlichen Parteien orientieren sich auf die deutsche bzw. jeweils nationale Sprache, geteilte Werte und sozialen Zusammenhalt und nicht auf erleichterten Zugang zu Staatsbürgerschaft, politische oder kulturelle Rechte. Leistungen am Arbeitsmarkt und in der Gesellschaft befähigen neu Zugewanderte erst, zu einem gedeihlichen Zusammenleben beizutragen.

Diese Überbelichtung von kulturellen Bedrohungen und die Missachtung von realen sozialen Problemen, die einer mangelnden oder gar fehlenden Politik im Umgang mit der Tatsache Vielfalt über Jahrzehnte geschuldet sind, sorgt dafür, dass die ungerechte Verteilung von Ressourcen, mangelnde Anerkennung und fehlende politische Mitsprache - wesentliche Elemente von Gerechtigkeit (Fraser 2007) - nach wie vor kaum angesprochen werden. Angebliche Bedrohungen, die von den zugewanderten Minderheiten ausgehen, berechtigen die Politik, Forderungen nach Anpassung unter dem Titel Integration in den Mittelpunkt zu rücken. Die Geschlechtergleichheit, die Anerkennung von Lesben und Schwulen, Respekt vor Demokratie und Diversität werden dabei zu den Aushängeschildern westlicher Demokratien erhoben.

Die Angst und die Angstmacherei vor „kulturellen Bedrohungen“ führen zu Forderungen nach einem Ende der multikulturalistischen Zugeständnisse an ethnische und religiöse Minderheiten, um soziale Segregation, Radikalisierung und Gewalt an Frauen zu bekämpfen. Die Art und Weise, wie dieser Schutz durch Anpassungsleistungen, Kontrolle und Messen als Förderung von Autonomie wie auch als Gewährleistung von Sicherheit repräsentiert wird,

\footnotetext{
${ }^{7}$ Wenn bei den islamkritischen Feministinnen das Engagement für den kleinen Mann auf der Straße fehlt, dann nur weil sie ihren Fokus auf die Naivität des Multikulturalismus richten und sich in ihrer Politik auf die Gefahren des Islam und der Muslime konzentrieren.
} 
bildet die Grundlagen für den „repressiven Liberalismus“. Mit diesem Begriff weist Christian Joppke zurück, dass wir es bei den jüngsten post-multikulturalistischen Entwicklungen mit verbesserten Formen der Inklusion zu tun hätten. Er schreibt die Spannungen auch nicht dem Nationalismus oder Rassismus, sondern dem Liberalismus und seinen illiberalen Mitteln selbst zu.

Eine Sonderstellung in der Liste der Bedrohungen, die mit Forderungen nach mehr Autonomie für Frauen in zugewanderten Kontexten beantwortet werden, kommt den so genannten „Kulturdelikten“ zu, die auch als „Gewalt im Namen der Ehre“ oder „traditionsbedingte Gewalt“ bezeichnet werden. Die damalige österreichische Innenministerin Maria Fekter (Österreichische Volkspartei) bezeichnete mit Kulturdelikten „Taten, die nach österreichischen Gesetzen illegal sind, bei Ausländern [sic!] aber zur Tradition gehören.“ Fekter hielt weiter fest, dass es notwendig sei, dass man die Dinge beim Namen nennt und den Zugewanderten klar macht: "Wie ist unsere Wertordnung und woran müssen sie sich halten“ (Die Presse, 7. August 2012). Diese „Delikte“ umfassen vor allem weibliche Beschneidung (FGM/C $)^{8}$, Ehrenmorde, Zwangsheirat und Kinderheirat und rufen damit den Kampf gegen Gewalt an Frauen und Jugendlichen „der anderen“ auf den Plan. Die kulturelle Beweisführung mittels der Differenzierungsmerkmale Geschlechteregalität und sexuelle Autonomie gegen „Türken“ und „Muslime“ als frauenfeindlich und homophob wurde zu neuralgischen Punkten der grundsätzlichen Kritik am Multikulturalismus. Seit Ehrenmorde und Zwangsverheiratungen unter dem Stichwort ,traditionsbedingte Gewalt“ verstärkt öffentlich thematisiert werden, werden Minderheiten homogenisiert und der Frauenfeindlichkeit bezichtigt. Sogar BefürworterInnen von multikulturalistischer Politik werden dadurch verunsichert, ob die mangelnde Durchsetzung von Menschen- und Frauenrechten (im Namen der Kultur) nicht ein grundsätzliches Überdenken von kulturellen Zugeständnissen erforderlich mache (Phillips 2007).

Welche Initiativen rechnet man nun in der Wissenschaft dem Multikulturalismus zu, welche Schwächen wirft man ihm aus welcher Perspektive vor und welche Ereignisse werden für den backlash, die rhetorische Ablehnung oder den repressiven Liberalismus verantwortlich gemacht?

\footnotetext{
${ }^{8}$ Weibliche Beschneidung wird in der politischen und wissenschaftlichen Debatte entweder Female Genital Mutilation (FGM) genannt, wenn die Betonung auf die Qual und die notwendige Bekämpfung der Gewalt gelegt wird, während andere die weniger moralisch aufgeladene Formulierung Female Genital Cutting (FGC) verwenden (Saharso 2008, S.12-13).
} 


\section{Wissenschaftliche Konjunkturen und Karikaturen des Multikulturalismus}

Im Gegensatz zu einem kommunitaristischen oder Differenzmultikulturalismus (Taylor 1993), der stärkeren Spielarten der Anerkennungspolitik mit kultureller Differenz und Gruppenrechten, beschränkt sich ein liberal-multikulturalistischer Ansatz (Kymlicka 1995, S. 35-44)) nicht auf kulturelle Anerkennung, sondern zielt auch auf den Schutz des Individuums. Zugewanderte Minderheiten dürfen demnach zwar die Macht der Mehrheiten begrenzen, um so den erwünschten Erhalt ihrer Institutionen sicherzustellen (external protection), sie dürfen dabei jedoch nicht die Rechte der einzelnen innerhalb der Gruppe einschränken (internal restriction). Diese Form des Multikulturalismus übt zudem Kritik an ökonomischen Ungerechtigkeiten und verlangt politische Repräsentation und die Teilnahme aller BürgerInnen auf allen Ebenen der Gesellschaft (Kymlicka 2010). Folgt man diesen „,schwachen“ ${ }^{\text {“9 }}$ Multikulturalismus, so umfasst diese Politik auch die Förderung des Zusammenlebens und den Zusammenhalt unterschiedlicher ethnischer und religiöser Gruppen, da die kulturelle Anerkennung dieser das Selbstbewusstsein auch der Individuen stärkt, wodurch Diskriminierung verhindert und Autonomie gestärkt werden sollen. Das Ziel dieser Politik ist eine gerechtere Teilnahme aller an allen Bereichen des Lebens und die Vermeidung, Entscheidungen einer bevorzugten nationalen Gruppe zu überlassen. Im Gegensatz zu einem monokulturellen Politikverständnis mit Assimilationserwartungen bewertet man aus einer multikulturalistischen Perspektive die Förderung von Unterschieden als fruchtbare Grundlage für eine gerechtere Gesellschaft. Gerechtigkeit durchzusetzen, bedeutet dann, dass politische Gemeinschaften auf allen Ebenen (lokal, regional, national und global) den Multikulturalismus annehmen und Maßnahmen in seinem Sinne umsetzen. Insbesondere der liberale Multikulturalismus beansprucht die Orientierung an Menschenrechten, Gleichheit und Freiheit, mit besonderer Berücksichtigung von Rechten und Angeboten, die Zugewanderten die Teilnahme an allen Bereichen der Gesellschaft und den Zugang zu Institutionen ermöglichen. Zugeständnisse an minorisierte Gruppen und Individuen sowie differenzierte, nicht homogenisierende Konzeptionen von citizenship sollen historisch gewachsenen Ungleichheiten entgegen wirken (Vertovec und Wessendorf 2010, S. 3; Kymlicka 2007, S.61ff und 2010, S.35f).

\footnotetext{
${ }^{9}$ Manche unterscheiden stark und schwach entlang der Forderung oder Ablehnung von Gruppenrechten, andere messen den Grad der Stärke an der Intensität des Engagements für diese Politik (Kymlicka 1995)
} 
Das Ziel dieser Politik ist demnach die Differenzierung und Vervielfältigung des Verständnisses von Bürgerschaft, sodass Minderheiten mit ihren Vorstellungen vom richtigen und guten Leben ausreichend Anerkennung bekommen, bei der Verteilung von Ressourcen berücksichtigt werden und die Möglichkeit erhalten, ihre Interessen in den politischen Foren zu vertreten. ${ }^{10}$ Die Menschenrechte bilden dabei durchaus einen Ausgangspunkt der Bestrebungen und nicht, wie oft von den „KarikaturistInnen des Multikulturalismus“ behauptet wird, einen Gegensatz. Der Multikulturalismus ist dieser liberalmultikulturalistischen Position nach Ausdruck differenzierter Gleichheit (Young 2007) und konzentriert sich insbesondere auf Fragen ethnischer und religiöser Minderheiten im Kontext von Kolonialismus, Nationenbildung oder Zuwanderung, schließt aber Ungleichheiten, wie sie (aus feministischer Perspektive) Frauen, sexuelle Minderheiten, Behinderte oder Alte erfahren und damit Ansätze der Diversität oder Intersektionalität von Machtfeldern nur teilweise mit ein.

Genau an den angepriesenen Stärken der multikulturalistischen Politik und an den expliziten Schwächen in Bezug auf Geschlechterverhältnisse setzt die Kritik ein. Der differenzblinde und auf Gleichheit orientierte Liberalismus bewertet alle differenzierten Zugänge zu Vielfalt ob multiple Unterdrückungsformen (Young 1989), Anerkennungspolitik (Taylor 1993), Versionen des Multikulturalismus (Kymlicka 1995, Parekh 2000) oder des multikulturellen Feminismus (Phillips 2007, Phillips und Saharso 2008) - als unvereinbar mit liberalen Grundsätzen und hält sie aufgrund der gesellschaftlichen Erfahrungen von Gewalt (wie 9/11, rassistisch wie auch islamistisch motivierte Morde) und von ungewollten sozialen Segmentierungen (hohe Zahl von SozialhilfeempfängerInnen und Arbeitslosen unter Zugewanderten) für überholt. Alle oben genannten multikulturalistischen Ansätze würden dem Grundgedanken liberaler Konzepte von citizenship entgegenstehen, vor allem den Prinzipien von Gleichheit, Freiheit und Solidarität. Obwohl der Multikulturalismus angetreten ist, die Gesellschaft angesichts ethnischer und religiöser Vielfalt zusammenzuhalten, geben ihm kritische Stimmen genau wegen dieser Betonung kultureller Identität nun die Schuld an der sozialen Segregation und sozio-ökonomischen Marginalisierung von Minderheiten. Anerkennung von kulturellen Differenzen führt zu Überbetonung und Unvereinbarkeit von ethnischen Gruppen mit unterschiedlichen Kulturen und mündet schließlich in sozialer

\footnotetext{
${ }^{10}$ Kymlicka (2010, S.36) bezeichnet dieses Bestreben und den Prozess für mehr Inklusion als citizenization.
} 
Segregation oder so genannten Parallelgesellschaften. Brian Barry behauptet sogar, dass der Multikulturalismus die Politiken der Umverteilung unterminiere (Barry 2001, S.8) ${ }^{11}$.

In letzter Zeit mehren sich Stimmen, wonach Anreize durch den Wohlfahrtsstaat und zu geringe Integrationsforderungen gegenüber Minderheiten zumindest mangelnde Partizipation am Arbeitsmarkt, soziale und räumliche Segregation und hohe Kriminalität zu verantworten hätten (Scheffer 2000; Wikan 2002; Koopmans 2008). Normativ sei ein multikulturalistischer Ansatz zwar vorzuziehen, in der Praxis habe er sich aber nicht bewährt.

„If there is one thing the European experience teaches us, it is that in dealing with the complex issues of difference and equality in immigration societies we cannot simply assume that what is normatively preferable from a rights-focused point of view will also be practically efficient from an outcomes point of view.” (Koopmans 2008, S.30)

Nicht nur liberale Ansätze und empirische Evaluierungen kritisieren den Multikulturalismus. Auch die linke politische Philosophie von Slavoj Žižek (2008) wendet sich gegen die Betonung von Differenz. Er verlangt vor allem nach Solidarität, die der Multikulturalismus, folgt man seinen AnhängerInnen, durch seine Betonung kultureller Unterschiede untergrabe. Diese Verschiebung von Solidarität zu Differenz würde nämlich die Kritik an der politischen Ökonomie durch partikuläre Interessen schwächen und Gewalt durch die Betonung von Ethnizität und Religion hervorbringen. Bemühungen um kulturelle Differenzen und AntiDiskriminierung verkleiden, naturalisieren und neutralisieren Ungleichheit und Ausbeutung. Toleranz wird als ,post-politischer“ Ersatz für Solidarität angesehen und für die Entpolitisierung des Sozialen verantwortlich gemacht (Žižek 2008, S. 660). Wenn Nationalismen und ethnisches Bewusstsein an Bedeutung gewinnen, wird der Wohlfahrtsstaat schwinden und neue Konflikte und Grenzziehungen werden immer wieder hervorbrechen.

Der Multikulturalismus hat demnach dem neoliberalen Kapitalismus nichts entgegenzusetzen, im Gegenteil verstärkt er diese Entwicklung und wirkt sogar selbst rassistisch, indem er Differenz absolut setzt und Angst vor Vermischungen schürt (Grillo 2003, S.164).

Auch wenn die Instrumentalisierung von kulturellen Differenzen zu wesentlichen Fallstricken multikulturalistischer Politik werden können, bleibt die Frage, wie Anerkennung von

\footnotetext{
${ }^{11}$ Barry wendet sich auch entschieden gegen Ausnahmeregelungen. So vergleicht er beispielsweise das Zugeständnis ritueller Schlachtungen polemisch mit seinem eigenen ,teuren Geschmack“, wonach er nur Fleisch von Tieren esse, die artgerecht gehalten und getötet würden (Barry 2001, S. 40). Derartige Vorlieben seien zwar berechtigt, bedürfen aber keiner gesetzlichen Unterstützung. Im Gegenteil ist er grundsätzlich überzeugt, dass »either the case for the law [...] is strong enough to rule out exemptions, or the case that can be made for exemptions is strong enough to suggest that there should be no law anyway (Barry 2001, S. 39).
} 
unterschiedlichen Erfahrungen und historisch gewachsenen Ungleichheit unter gegebenen Machtverhältnissen ${ }^{12}$ sinnvoll vor differenzblinden Lösungen des Anti-Multikulturalismus geschützt werden können. Christian Joppke meinte 2004 dazu, dass Norman Glazers Ausspruch „we are all multiculturalists now“ ohnehin nach wie vor Gültigkeit hätte und dass kein westlicher Staat seinen BürgerInnen eine kulturelle Identität aufzuzwingen versuche. Es handelt sich jedoch seiner Ansicht nach um eine ent-ethnisierte oder post-ethnische Forderung nach liberaler Demokratie mit der Betonung auf Menschenrechten und Gleichheit im Aufnahmeland unter dem Stichwort civic integration. Nach Joppke bleiben westliche Gesellschaften trotzdem plural und legen Wert auf Respekt vor Diversität (Joppke 2004, S. 254f.).

"What then is the retreat from multiculturalism? Perhaps a growing sense of "when in Rome do as the Romans do' has not been outlived as a maxim of immigrant integration, precisely because the contemporary >Romes are polyglot places in which the ties that bind are increasingly procedural and universalistic.” (Joppke 2004 S. 255)

In den letzten Jahren identifiziert Joppke (2007) jedoch Repressionen durch den Liberalismus, die nicht als Rassismus oder Nationalismus erklärt werden können, sondern eine liberale Antwort auf die Integrationskrise oder den Multikulturalismus-Backlash darstellen würden. Aufgrund des größten sozio-ökonomischen Integrationsfehlschlags in Europa, den er mit Ruud Koopmans (2010) in den Niederlanden ortet und mit hohen Zahlen von WohlstandshilfeempfängerInnen, exkludierenden Arbeitsmärkten, segregierten Wohngegenden und hohen Kriminalitätsraten verbindet, werden Initiativen zur ,zivilen Integration“" gestartet. Das Ziel sind integrierte BürgerInnen, die zivile Werte wie Gleichheit, Freiheit und Demokratie teilen. Zu Beginn war das Ziel dieser neuen Integrationsinitiative, Zugewanderten rasch den Weg in die Institutionen des Aufnahmelandes zu zeigen und ihnen damit „Autonomie“ zu gewähren (übrigens ein zentrales Anliegen des liberalen Multikulturalismus). Im Laufe der Zeit wurde jedoch deutlich, dass die Maßnahmen dafür (Sprachkurse vor der Einreise, Integrationsvereinbarungen mit Sprach-, Landeskunde- und Staatsbürgerschaftstests) nicht nur verpflichtend und bei Nichterfüllung mit Sanktionen

\footnotetext{
${ }^{12}$ Iris Marion Young beschreibt in ihrer Auseinandersetzung mit der Politik der Differenz den Wechsel von positional difference zu cultural difference in den 1990er Jahren auch als cultural turn. Während die positionale Differenz der feministischen, anti-rassistischen und Lesben/Schwulen Bewegungen strukturelle Gleichheit einfordert, zielt eine Politik der kulturellen Differenz (aufgrund von Nationalität, Ethnizität oder Religion) auf den Wert der Förderung kultureller Unterschiede, und dies als kompatibel mit Gerechtigkeit (Young 2007, S. 60ff). Für eine Vertiefung dieser Politik geht es auch um eine gemeinsame Behandlung dieser zwei Achsen von Politik der Differenz, da sie beide differenzblinde Positionen als Teil des Problems und nicht der Lösung identifizieren.
} 
verbunden waren, sondern dass im Laufe der Zeit zunehmend das Problem von Leistbarkeit und Machbarkeit privatisiert wurde. Integrationsinitiativen werden dadurch mit Immigrationskontrolle vermischt und zu einem Mittel der Ausgrenzung. Die von der Politik immer explizit genannten oder implizit gemeinten Zielgruppen dieser „,neuen“ oder intensiveren Maßnahmen sind in den unterschiedlichen Ländern Europas wenig gebildete Muslime, egal ob türkischer, marokkanischer, algerischer oder pakistanischer Herkunft. Ohne weitere Reflexion werden sozio-ökonomische und bildungspolitische Probleme somit exportiert, zumindest aber aus der institutionellen Verantwortung der Einwanderungsländer ausgelagert.

Während die dominante Politik in den EU Mitgliedsstaaten bemüht ist, durch Integrationsinitiativen Komplexität besser zu verwalten sowie Kriminalität, Marginalisierung und Gewalt einzudämmen, streben gegenhegemoniale Bewegungen wie auch manche kulturund sozialanthropologische Beiträge (auch im deutschsprachigen Raum) danach, den Integrationsimperativ selbst kritisch auszuleuchten.

\section{Sozialanthropologische Beiträge zum Multikulturalismus-Streit}

In allen Diskurssträngen um den Multikulturalismus gerät seit vielen Jahren immer wieder der Kulturbegriff ins Zentrum der Aufmerksamkeit. Sozial- und kulturwissenschaftliche TheoretikerInnen haben längst Ansätze zur Verfügung gestellt, um Gleichheit und Differenz wie auch soziale Umverteilung und kulturelle Anerkennung zusammenzuführen und Komplexitäten der spätmodernen Gesellschaft in den Konzepten wie Transnationalität (GlickSchiller 1992 et al., 1995 et al., 2009), Intersektionalität (siehe Yuval-Davis 2006) oder Kultur als duale diskursive Praxis (Baumann 1996, 1999) zu fassen. Trotzdem wurde dem Multikulturalismus ein essentialistischer und deterministischer Kulturbegriffs unterstellt, der erst den Vorwurf der Kulturalisierung sozialer Probleme ermöglichte. Verursacht durch diese Konstruktionen von Kultur erschien der Multikulturalismus dann für trennende und sozial marginalisierende Wirkungen verantwortlich und seine Zurückweisung (zumindest rhetorisch) unausweichlich (Koopmans 2010).

Die Sozial- und Kulturanthropologie beschäftigt sich während dieser Debatten für und wider den Multikulturalismus einerseits mit der Vervielfältigung von Differenzen in den europäischen Gesellschaften mit Hilfe von Konzepten wie Super-Diversität (Vertovec 2007), 
mit dem Verlassen des methodologischen Containerdenkens durch transnationale Ansätze (Wimmer und Glick-Schiller 2002) und mit alternativen Politiken wie der Kosmopolitisierung „,von unten“ (Römhild 2009). Zudem werden in der sozialanthropologischen

Migrationsforschung post-ethnische Ansätze diskutiert (Glick-Schiller und Cağlar 2011), die sich von der dominanten Frage nach Recht und Gerechtigkeit abwenden und den jeweiligen Bedeutungen von MigrantInnen im Zuge von städtischen Transformationen nachgehen.

Neben diesen grundlegenden konzeptuellen Neuorientierungen gibt es lange Zeit bereits auch sozialanthropologische Diskussionen zu den Ambivalenzen der Disziplin (Turner 1993; Eller 1997) zur Politik des Multikulturalismus (bestätigend oder kritisierend) beizutragen. In den letzen Jahren gab es allerdings durchaus eindeutig kritische Interventionen zur „Integration neu“ im deutschsprachigen Raum. Sowohl durch „genaues Hinsehen“ (Schiffauer 2008) auf die Bedrohungsszenarien des Anti-Multikulturalismus (wie Ehrenmorde, Moscheenvereine, Geschlechterverhältnisse) wie auch durch kritische Analysen des neuen Integrationsparadigmas (Hess et al. 2009; Sezgin 2011; Strasser 2010, 2012), meldeten sich Sozial- und KulturanthropologInnen zu Wort. Insbesondere die Konzepte zu Kultur oder genauer zu deren Praxis im Zusammenhang mit Essentialismus und Fundamentalismus sollen hier noch einmal kurz zusammengefasst und in ihrer möglichen Bedeutung für die Politik beleuchtet werden.

Wenn dem Multikulturalismus ein starrer und unveränderbarer Kulturbegriff zu Grunde gelegt wird, verliert diese Politik das Potential dynamische kultursensible Lösungen für Gerechtigkeitsfragen zu entwickeln und ist selbst in Gefahr zu Grenzziehungen beizutragen statt diese zu überwinden. Essentialistische Kulturauffassungen bilden somit die Voraussetzung, Kultur und Tradition der Anderen als importierte und unveränderbare Zwangsjacke (Cağlar 1990) und damit als das zentrale Problem darzustellen. Der Multikulturalismus wird durch die Annahme, er würde die starre Kultur der Minderheiten fördern, für Parallelgesellschaften und soziale Marginalisierung verantwortlich gemacht. Bevor jedoch das (multi-)kulturelle Kind mit dem kulturalistischen Bade ausgeschüttet wird (Grillo 2003, S.164), sollte die Möglichkeit angedacht werden, einen Multikulturalismus ohne kulturellen Essentialismus zu entwickeln.

In diesem Sinn weist die feministische Politikwissenschafterin Anne Phillips, wie zuvor schon Ayşe Cağlar (1990), auf die Gefahr von „cultural straitjackets“ (Phillips 2007, S.13) hin und argumentiert wie schon andere vor ihr, dass eigentlich der Essentialismus dem Ziel der 
multikulturalistischen Politik im Weg steht und nicht die Kultur. Sie fragt jedoch dann weiter: "[I]f culture imputes a false stability to experiences that are intrinsically fluid, what exactly is left to be recognised, accommodated, or equalised?" (Phillips 2007, S.14). Sie sieht für dieses Problem weder im Anti-Essentialismus noch im Konstruktivismus eine Lösung, sondern schlägt einen besonders herausfordernden Umgang mit Kultur vor.

,[A] multiculturalism that dispenses with the reified notions of culture that feed those stereotypes to which so many feminists have objected, yet retains enough robustness to address inequalities between cultural groups, a multiculturalism in which the language of cultural difference no longer gives hostages to fortune or sustenance to racists, but also no longer paralyses normative judgment." (Phillips 2007, S.8)

Sozialanthropologische Theorien suchen insbesondere seit der postkolonialen Kritik an der festschreibenden Repräsentation aufgrund ihres kulturrelativistischen Ansatzes ebenfalls nach Wegen zwischen Gefahren des Kulturalismus einerseits und postkolonialen Dekonstruktionen andererseits. Je deutlicher allerdings der Konsens unter TheoretikerInnen von Kultur als historisch entstanden, vielstimmig, stets im Wandel begriffen, im Inneren heterogen und nach Außen durchlässig ausfiel, desto weniger ließen sich die AkteurInnen in den Debatten zum „Kulturkonflikt“ von der Harmlosigkeit der Kulturen überzeugen. Viele SozialwissenschafterInnen wandten sich im Zuge dieses zunehmenden kulturellen Unbehagens vom Kulturbegriff ab. Andere hingegen wandten sich explizit der Untersuchung des kulturellen Essentialismus zu: „If people are doing this thing called bounding and closure and essentialism, should this not be recognized as a real social phenomenon rather than shunned as a terrible mistake?“ (Friedman, 2003, S.30).

Wenn die Sozialwissenschaft das „,kulturelle Unbehagen“ (Grillo 2003) in einer Gesellschaft besser verstehen will, das wesentlich aus der Angst vor dem Verlust der eigenen Werte und in Ablehnung der anderen „Kulturen“ besteht, müssen die gesellschaftlichen Hintergründe der kulturellen Zurückweisungen mitgedacht werden. Es geht im Kern wohl um die Frage, ob Kultur als Merkmal der Unterscheidung ein gegenseitiges Verstehen verhindert, oder ob die angeblich unveränderbaren Unterschiede erst das Ergebnis der erwünschten Grenzziehung darstellen, um soziale und ökonomische Ungleichheiten zu legitimieren. Prozesse der Grenzziehungen und das Zusammenspiel von essentialisierenden und abweichenden oder nach Baumann dominanten und demotischen Diskursen und Praktiken (Baumann 1996) von Kultur bilden aus sozialanthropologischer Sicht wesentliche Bausteine, um Identitätsprozesse 
und Grenzziehungen in von Diversität gekennzeichneten Gesellschaften zu verstehen. Die spezifischen Ausprägungen von „Kultur als Essenz“ müssen demnach, genau wie Abweichungen im jeweiligen Kontext und in ihrer Entstehungsgeschichte untersucht werden. Der Blick auf die Prozesse von Essentialisierungen und Abweichungen ermöglicht somit alle daran Beteiligten einzubeziehen sowie die Herstellung von Gruppen und Grenzen selbst zum Untersuchungsgegenstand zu machen (Barth 1994; Baumann 1999).

Empirische und theoretische Befunde (Baumann 1996 und 1999; Grillo 2003) zeigen, dass Essentialisierungen unvermeidbare Erfahrungen in sozialen Prozessen darstellen, die als Grenzziehungen neben Transformationen und Abweichungen nicht einfach zurückgewiesen werden können, sondern in ihrer Komplexität untersucht werden müssen. Beziehungen und damit Konflikte zwischen Menschen, die sich um Rechte, Anerkennung, Umverteilung und Partizipation bemühen, verändern sich nicht, weil kulturelle Muster von der Wissenschaft oder anderen außenstehenden BeobachterInnen dekonstruiert werden. Essentialismen lassen sich zudem aus der Perspektive der ProtagonistInnen in der alltäglichen Praxis nicht leicht als solche erkennen und für die Identifikation mit politischen Kämpfen nicht einfach durch alternative und bewegliche Sichtweisen ersetzen (Benhabib 1999). Sind es zudem nicht letztlich die Essenzen oder zu Wahrheit gewordene Erzählungen, die Staaten legitimieren, Kindererziehung erleichtern und Zugehörigkeiten vermitteln?

Der Blick auf die Praxis ermöglicht somit Essentialisierung als kulturellen Prozess zu fassen, der alle beteiligten AkteurInnen auf lokaler, transnationaler und globaler Ebene einbezieht. Dadurch wird die Relevanz von globalen Menschenrechten und partikulären kulturellen Vorstellungen genauso wie deren jeweilige Bedeutungen in den alltäglichen Praktiken von minorisierten und dominanten AkteurInnen zum Untersuchungsgegenstand. So wird das sozialanthropologische Fleisch zum rechtlichen und politischen Skelett der Gerechtigkeit hinzugefügt (Hastrup 2002), so werden Wirkungen und Verhandlungen von Rechtsfragen und staatlichen Institutionen im Alltag aller Beteiligten sichtbar gemacht. SozialanthropologInnen unterscheiden für die Untersuchung dieser lokalen oder transnational verflochtenen Prozesse einen „deskriptiven Kulturrelativismus“ von normativen und absoluten Begriffen von Relativismus. Der deskriptive Relativismus ist demnach nicht mehr als ein Werkzeug der WissenschafterInnen, das es ermöglicht, Perspektiven der untersuchten Individuen 
weitgehend ohne ethnozentrische Vorannahmen nachzuzeichnen oder eben ,genau hinzuschauen“ (Schiffauer 2008). ${ }^{13}$

Dieses genaue Hinsehen mit einem konventionellen Relativismus ermöglicht Informationen über alltägliche Erfahrungen verlangt aber auch eine Pendelbewegung (Dembour 2001) zwischen „genauem Hinsehen“ und „kritischem Positionieren“. Insbesondere um verletzbare Subjekte vor Gewalt schützen zu können, genügt das Verstehen nicht, sondern bildet lediglich die unabdingbare Voraussetzung, um zwischen partikulären Vorstellungen und universellen Rechten eine möglichst gut informierte Position ergreifen zu können.

\section{Multikulturelle Verletzbarkeit: Feministische Debatten}

Eine besondere Variante des Streits um den Multikulturalismus stellt die feministische Debatte dar. Susan Okins (1999) provokante Frage, ob der Multikulturalismus Frauen zusätzlich benachteiligen würde und damit schlecht für Frauen sei, löste heftige Kontroversen unter feministischen WissenschafterInnen aus. Nach Okin schränkt die Durchsetzung von Rechten für Minderheiten die Autonomie von Individuen mit abweichenden Meinungen innerhalb von diesen ethnischen oder religiösen Gruppen ein. Daher ist aus ihrer liberal feministischen Perspektive eine Kritik an den blinden Flecken multikulturalistischer Theorien und Politik unumgänglich. Unni Wikan (2002), ebenfalls dem liberalen Flügel zuzuordnen, sprach in diesem Zusammenhang sogar von ,großzügigem Verrat“" an jungen Frauen, denen im Namen der Anerkennung von Kultur Unterstützung durch westlich-liberale Staaten beim Kampf um Freiheit versagt bliebe. Die gleichzeitige Forderung nach dem Recht auf Differenz und dem Schutz von „Minderheiten innerhalb von Minderheiten“ oder gefährdeten Individuen wie Frauen, Lesben und Schwule begründen die Schwäche des Multikulturalismus, oder die „mutlicultural vulnerabilty“ (Shachar 2000).

\footnotetext{
${ }^{13}$ Immer wieder wird dieser schwache oder konventionelle Relativismus allerdings in normative oder sogar epistemologische Versionen verwandelt. Der normative Relativismus verweist auf die Unmöglichkeit, eine andere Kultur aufgrund universeller Standards zu beurteilen, wodurch jegliches Wissen und Handeln als kulturabhängig betrachtet und jeglicher Kritik von außen unzumutbar wird. Diese Form des Relativismus bildet das partikularistische Gegenüber in den Positionskämpfen um universelle Menschenrechte. Der epistemologische Relativismus geht sogar so weit, jegliche verallgemeinernde Theorie von Kultur oder Möglichkeiten des Vergleichs zwischen Kulturen abzulehnen. Da dieser Versuch, alle Kulturen als gleich wertvoll aber unverstehbar zu sehen, auch unüberbrückbare Unterschiede postuliert, kann er nicht zur Lösung von Problemen in einer multikulturalistischen Gesellschaft beitragen, sondern führt im Gegenteil zu „Kulturalismus“ oder einem „Rassismus ohne Rasse“ (Balibar 1990).
} 
Im Zentrum der feministischen Debatten um die Zurückweisungen des Multikulturalismus in der EU standen die Morde an Fadime Şahindal 2003 (Schweden) und Hatun Sürücü 2005 (Deutschland) und (höchst emotionale) Berichte von jungen Frauen über ihr Leben mit Zwang und Gewalt im Inneren ethnischer und religiöser Minderheiten (Necla Kelek, Ayaan Hirsi Ali, Sabatina Jones). Das darum entstandene Medienereignis und eine große Zahl an Konferenzen und Initiativen heizten Vermutungen über hohe Dunkelziffern von Ehrenmorden, Zwangsverheiratungen und Genitalbeschneidungen weiter an. Globale und nationale Initiativen gegen Gewalt im Namen der Ehre, Expertinnen-Meetings, wissenschaftliche Studien und neue gesetzliche Regulierungen sollten diese Phänomene der traditionellen oder auf Kultur basierenden Frauenunterdrückung in zugewanderten Minderheiten bekämpfen (Schiller 2010; Strasser und Holzleithner 2010).

Zwangsehe wurde medial besonders umfangreich diskutiert und in vielen Ländern unter Strafe gestellt. Die Erhöhung des Strafausmaßes für die Verantwortlichen bei Zwangsehen und die Ausdehnung des Kreises der Beschuldigten stellte sich bald als unwirksam heraus (Beclin 2010). Da junge Frauen ihre Familie selten vor Gericht zerren (schon gar nicht, wenn Gefängnisstrafen drohen) beweist dieser Misserfolg natürlich nicht, dass es keinen Zwang bei Eheschließungen gibt. Es zeigt nur, dass präventive Maßnahmen sinnvoller sind und dass Schutzeinrichtungen installiert werden müssen, damit Mädchen und Frauen, aber auch Burschen, die aus einer erzwungenen Ehe fliehen wollen, eine Unterkunft angeboten werden kann. Doch trotz der hohen medialen Aufmerksamkeit und dem oftmals ausgesprochenen politischen Willens fehlen effektive Maßnahmen in den meisten europäischen Ländern bis heute. Am effektivsten schützte das multikulturelle England junge Frauen, annullierte viele Ehen und holte von Zwangsehe Betroffene auch aus anderen Ländern zurück, in die sie verheiratet worden waren, ohne die Zwangsehe unter Strafe zu stellen (Phillips und Dustin 2004; Phillips 2007, 2010). Das neue Integrationsparadigma unter dem konservativen Premierminister David Camaron läßt jedoch befürchten, dass dort ebenfalls und gegen den Willen der zuständigen Behörden eine Kriminalisierung erfolgt (BBC News 5. Februar 2011). Auf jeden Fall bildeten diese Formen von „Gewalt im Namen der Tradition“ ein gewichtiges Argument für die Forderungen nach dem Rückzug vom Multikulturalismus.

Kultur wurde in diesen Debatten, unabhängig von der komplexen wissenschaftlichen Debatte zu diesem Begriff seit den 1990er Jahren, eine essentialisierende und determinisierende Wirkung zugesprochen. Bezeichnungen wie „Gewalt im Namen der Tradition“ oder „kulturbedingte Gewalt“ weisen auf diese enge Verbindung von Gewalt mit Kultur oder 
genauer gesagt auf den Vorwurf, dass manche Kulturen „gewaltvoll“" seien und sich deshalb entweder verändern oder auflösen sollten (Okin 1999). Liberale Feministinnen stärkten somit den Ruf nach gemeinsamen Grundwerten und neuen Integrationsinitiativen.

In der folgenden ebenfalls feministischen Kritik an den liberalen Positionen wurde auch verlangt, dass Frauen, Kinder, Alte und sexuelle Minderheiten genauso wie minorisierte nationale, ethnische oder religiöse Gruppen besonders beachtet und vor jeder Form von Gewalt geschützt werden müssen. Doch gleichzeitig verlangte diese Position, dass dieser Schutz nicht den Rückzug von dem Recht auf Differenz bedeuten dürfe und kulturalistische Zuschreibungen vermeiden müsse. Es mehrten sich also die Stimmen, die homogenisierende und abwertende Diskurse „im Namen der Geschlechteregalität“ als strukturelle Gewalt kritisierten und versuchten, einen sich ausbreitenden Generalverdacht gegen Zugewanderte, Neuzuwanderung und Diversität zu verhindern (Volpp 2001; Razack 2004; Eisenberg und Spinner-Halev 2005; Phillips 2007; Strasser und Holzleithner 2010).

"Multiculturalism became the scapegoat for an extraordinary array of political and social evils, a supposedly misguided approach to cultural diversity that encouraged men to beat their wives, parents to abuse their children, and communities to erupt in racial violence." (Phillips 2007, S.3)

Vielen feministischen Multikulturalistinnen wurde es unter den Bedingungen des AntiMultikulturalismus ein Anliegen, einen produktiven Umgang mit der schwierigen Beziehung zwischen zentralen Begriffen dieser Debatte zu entwickeln. Viktimisierungen von Frauen aus minorisierten Kontexten wurden genauso hinterfragt wie kulturelle Homogenisierungen und gesetzliche Normierungen zur Bekämpfung von Gewalt gegen Frauen „im Namen der Ehre“. Neben sozialer Gleichheit und kultureller Differenz wurde auch das Verhältnis zwischen Kultur und Autonomie diskutiert. Dabei muss der Kulturbegriff ebenso wie jener der Autonomie angemessen formuliert werden.

„Nur auf Grundlage einer Konzeption von Kultur, die deren prozesshaften Charakter ebenso einfangen kann wie die Tendenz, Kultur im Alltag zu essentialisieren und zu politisieren, kann ein brauchbarer normativer Vorschlag entwickelt werden, der die Anliegen der Geschlechtergleichheit und der sexuellen Autonomie in ihren jeweiligen kulturellen Kontexten annehmbar macht. Autonomie wird dabei selbst als eingebettet in und als Ausdruck von kulturellen Prozessen begriffen.“ (Holzleithner und Strasser 2010, S.27) 
Aus einer liberalen Perspektive wurde sexuelle Autonomie zunehmend zu einem unverzichtbaren Kennzeichen von Demokratie, während aus einer multikulturalistischen Perspektive Autonomie sich verstärkt als ein Mittel der Repression darstellte und damit als eine auf Geschlecht basierende Variante von „repressiven Liberalismus“ erschien. Die EURichtlinie (RL 2003/86/EG) zu Familienzusammenführung zeigt besonders deutlich, wie der Kampf gegen Gewalt an Frauen in einem Klima des Anti-Multikulturalismus zu Ausgrenzungen beiträgt und schließlich statt zu mehr Autonomie zu verstärkter Kontrolle von Frauen, transnationalen Ehen und Migration beiträgt. Um dem angeblich naiven Multikulturalismus Einhalt zu gebieten und Zwangsehen zu verhindern, wurde den Mitgliedsstaaten die Erlaubnis zur Einführung eines Mindestalters bei der Familienzusammenführung mit Drittstaatsangehörigen eingeräumt. Obwohl die entsprechende EU-Richtlinie Familienzusammenführung als wesentliche Voraussetzung von Integration bezeichnet, wird die Einführung eines Mindestalters von maximal 21 Jahren erlaubt.

„Zur Förderung der Integration und zur Vermeidung von Zwangsehen können die Mitgliedstaaten vorsehen, dass der Zusammenführende und sein Ehegatte ein Mindestalter erreicht haben müssen, das höchstens auf 21 Jahre festgesetzt werden darf, bevor der Ehegatte dem Zusammenführenden nachreisen darf.“ (Richtlinie 2003/86/EG, Artikel 4)

Auch wenn die Vereinbarkeit dieser Fakultativklausel mit der Europäischen Menschenrechtskonvention (Artikel 8 und 12) zumindest fraglich ist, haben Großbritannien, Frankreich, Norwegen und Dänemark seither Mindestaltersgrenzen zwischen 18 und 24 Jahren eingeführt. Durch diese Maßnahme werden jedoch nicht einzelne kriminelle Fälle von Zwangsverheiratung behandelt, sondern alle Drittstaatsangehörigen wenn nicht kriminalisiert, so doch in gravierender Weise benachteiligt. Dass diese Option nicht nur Zwangsehen verhindert, sondern generell transnationale Ehen erschwert und die Zuwanderung nach Europa verstärkten Kontrollen aussetzt, liegt auf der Hand. Damit bestätigt diese Maßnahme die vorherrschende medial vermittelte öffentliche Meinung in ihrer generalisierenden Annahme, dass ,importierte Traditionen“ von allen Angehörigen einer ethnischen Gruppe geteilt würden. Aus dem Schutz vor Zwangsehe wird somit eine handfeste indirekte Diskriminierung, die davon ausgeht, dass alle Jugendlichen der zugewanderten Minderheiten geschützt werden müssen. Diese Sichtweise von Kultur als deterministisch und die daraus abgeleitete Einschränkung von Optionen führt nicht zu mehr Autonomie von jungen Frauen, sehr wohl aber zu einem abnehmenden Vertrauen in die Behörden und Institutionen der europäischen Gesellschaften. 
Der Streit um den Multikulturalismus mündete gestützt auf Debatten um Demokratiefähigkeit, räumliche Segregation, Arbeitslosigkeit, Kriminalität und nicht zuletzt auf Gewalt gegen Frauen in einen Anti-Multikulturalismus und die Forderung nach einer neuen effektiveren Politik der Inklusion. Die EU Kommission reagierte mit einer Integrationsagenda (2005), die im Rahmen der Bekämpfung von Gewalt gegen Frauen und von Marginalisierung von Minderheiten zunehmend Regulierung von Migration betreibt, die jedoch zugewanderte oder zuwanderungswillige Frauen zunehmend unter den Verdacht von Zwang- und Scheinehe stellt. Der Kampf gegen Gewalt an Frauen verwandelte sich durch die gesetzten Maßnahmen und die dominanten Diskurse zu einem weiteren Beitrag des Liberalismus, der mit illiberalen Mitteln weitere Ungleichheit erzeugt. Statt Frauen mit mehr Freiheit und Selbstbestimmung auszustatten, werden sie einer „repressiven Autonomie“ unterworfen. Die Forderung nach Autonomie wird in ihrem Effekt repressiv, wenn sie statt Selbstbestimmung und Geschlechteregalität zu fördern, die Unterordnung von minorisierten Frauen gegenüber „einheimischen“ oder dominanten sicherstellt. Diese Wirkung erklärt das plötzliche und überraschend rasende Interesse von konservativen Parteien und patriarchalen Männern an Frauenrechten und dem Rückzug vom Multikulturalismus.

\section{Nach dem Multikulturalismus-Streit: Zusammenfassung und Ausblick}

Debatten zu räumlicher Segregation, steigender Arbeitslosigkeit oder bedrohter Sicherheit führten in den letzen Jahren immer wieder zu Forderungen nach dem Rückzug vom Multikulturalismus. Die multikulturalistischen Politik würde kulturelle Differenzen betonen und essentialisieren, Grenzziehungen verstärken und individuelle Autonomie untergraben. Kulturelles Unbehagen breitete sich zudem rasch mit rechtspopulistischer Politik zu den Themen Migration, Kriminalität und Geschlechteregalität „der anderen“ aus und wurde ebenfalls dem Multikulturalismus angelastet.

Die Abkehr vom Multikulturalismus entfaltet sich vor gleich mehreren verwobenen und nur schwer zu entwirrenden Trugschlüssen. Doch immer wieder wird ein starker Multikulturalismus statt eines schwachen Multikulturalismus herangezogen (Modood und Werbner 1997), um diesen dann wegen der Verdinglichung von Kultur, die angeblich zu den Barrieren zwischen Menschen beiträgt, besser kritisieren zu können. Die praktische Herausforderung ist jedoch nicht die kulturelle Differenz, sondern die Fixierung von Differenz, nicht Kultur, sondern der kulturelle Essentialismus verbunden mit der Angst vor 
Verlust von Identität durch kulturelle Vermischungen. Nicht die ethnisch-kulturelle

Perspektive, sondern der Kulturalismus bildet das Kernproblem des Multikulturalismus. Doch ein Anti-Essentialismus und der rhetorische Wechsel zu Integrationsansätzen oder zu Diversitätskonzepten (die ein dynamisches Konzept von Kultur beanspruchen) bewahren uns nicht vor der Einschränkung von Freiheit und Selbstbestimmung. Im Gegenteil mündet die Abkehr von der Politik der Anerkennung und die Förderung gemeinsamer Werte und Normen durch unterschiedliche Kursmaßnahmen und Tests wiederum in eine Politik, die Forderungen ausschließlich an zugewanderte Minderheiten richtet. Auch wenn die Forderungen Freiheit, Gleichheit und Demokratie genannt werden, unterstellen die Verantwortlichen der Einwanderungsländer damit, dass Zuwanderer diese nicht ohnedies teilen, sondern erst erlernen müssen. Sie wenden zudem Mittel an, die (wie im Fall von Neuregelungen der Familienzusammenführung genauso wie im Fall von Integrationsvereinbarungen durch Leistungstests) nicht zu mehr Freiheit, sondern zu mehr Kontrolle und Repression beitragen.

Seit den Debatten um Geschlechteregalität tummeln sich viele selbsternannte FeministInnen in konservativen Parteien und verlangen Gesetze gegen „Kulturdelikte“. Die lautstarke Debatte um Gewalt gegen Frauen führte schließlich 2003 zu einer EU-Direktive, die zwar nicht das Heiratsalter aber das Alter für Familienzusammenführung anzuheben ermöglichte. Viele Länder machten von dieser Möglichkeit Gebrauch und entscheiden sich für eine repressive Umgangsweise mit Ehen mit Drittstaatsangehörigen. Was Zwang verhindern und Freiheit fördern soll, stellt umgekehrt alle Ehen mit Drittstaatsangehörigen unter Generalverdacht und schränkt die Wahlfreiheit ein. Dieser Übereifer, der behauptet Frauen und Kinder Autonomie zu gewähren und dabei autonome PartnerInnenwahl verhindert, der nicht im Namen von Kultur, sondern im Namen von liberalen Werten und Gleichheit Gewalt ausübt, also demnach einen „Gleichheitsdelikt“ darstellt, diesen Prozess der „erzwungenen Freiheit“, der illegitime Mittel zur Durchsetzung von Freiheit anwendet, bezeichne ich als „repressive Autonomie“.

Liberalismus gemischt mit Leistungsanforderungen und verfehlten Freiheitsangeboten leitet derzeit die Integrationspolitik in Europa. Der Rückzug vom Multikulturalismus führt zu einer Integrationsdebatte, die Minderheiten nicht stärkt, sondern einer monokulturellen und neoliberalen Leistungsgesellschaft zuführt, die alle nicht Leistungsstarken ausschließt und folglich sein Ziel der sozialen Integration von Beginn an verfehlt. Auch wenn der Multikulturalismus nicht überall gleich erfolgreich war, in der hier untersuchten Frauenpolitik verstand das multikulturalistisch ausgerichtete England noch am besten Gewalt zu 
bekämpfen. Das wirft die Frage auf, ob nicht ein neo-multikulturalistischer Ansatz, der Differenz weder esssentialisiert, noch ihre Relevanz mit (überwiegend repressiven) Forderungen nach individueller Freiheit und sexueller Autonomie verdeckt, einer Politik der „Integration neu“, vorgezogen werden sollte.

\section{Literatur}

Balibar, E. 1990 [1988]. Gibt es einen „Neo-Rassismus“? In Rasse Klasse Nation. Ambivalente Identitäten, hrsg E. Balibar und I. Wallerstein, 23-28. Hamburg, Berlin: Argument Verlag.

Barry, B. 2001. Culture and Equality. An Egalitarian Critique of Multiculturalism. Cambridge (Massachusetts): Harvard University Press.

Barth, F. 1994. Enduring and emerging issues in the analysis of ethnicity. In The Anthropology of Ethnicity. Beyond Ethnic Groups and Boundaries, hrsg H. Vermeulen und C. Govers, 11-32. Amsterdam: Het Spinhuis.

Bauböck, R. 2002. Farewell to Multiculturalism? Sharing values and identities in societies of immigration. Journal of International Migration and Integration 3 (1): 1-16.

Bauböck, R. 1997. Migrationspolitik. In Handbuch des politischen Systems Österreichs, hrsg H. Dachs, P. Gerlich und H. Gottweis, 3. Überarbeitete Auflage, Wien: Verlag Manz.

Baumann, G. 1996. Contesting Culture. Discourses of Identity in a Multicultural Europe. Cambridge: Cambridge University Press.

Baumann, G. 1999. The Multicultural Riddle. Rethinking National, Ethnic and Religious Identities. New York, London: Routledge.

Beclin, K. 2010. Rechtliche und politische Strategien gegen Zwangsehen in Osterreich. In Multikulturalismus queer gelesen. Zwangsheirat und gleichgeschlechtliche Ehe in pluralen Gesellschaften, hrsg Strasser, S. und E. Holzleithner, 144-164. Frankfurt/Main: Campus. Benhabib, S. 1999. Kulturelle Vielfalt und demokratische Gleichheit. Politische Partizipation im Zeitalter der Globalisierung. Frankfurt am Main: Fischer. 
Brubaker, R. 2001. The Return of Assimilationism? Changing perspectives on immigration and its sequels in France, Germany, and the United States. Ethnic and Racial Studies 24: 531548.

Cağlar, A. 1990. Das Kultur-Konzept als Zwangsjacke in Studien zur Arbeitsmigration. Zeitschrift für Türkeistudien 1: 93-105.

D'Amato, G. 2010. Switzerland: a multicultural country without multicultural policies. In The multiculturalism backlash. European discourses, policies and practices, hrsg S. Vertovec and S. Wessendorf, 130-151. Oxon, New York: Routledge.

Dembour, M-B. 2001. Following the Movement of a Pendulum. Between Universalism and Relativism. In Culture and Rights. Anthropological Perspectives, hrsg J. K. Cowan und M-B. Dembour, and R. A. Wilson 56-80. Cambridge: Cambridge University Press.

Eller, J. D. 1997. Anti-Anti-Multiculturalism. American Anthropologist 99 (2): 249-260.

Eisenberg A. und J.Spinner-Halev (Hrsg.). 2005. Minorities within Minorities. Cambridge: Cambridge University Press.

Fraser, N. 2007. 'Re-framing justice in a globalizing world'. In (Mis)recognition, social inequality and social justice. Nancy Fraser and Pierre Bourdieu, hrsg T. Lovell 17-35, London: Routledge.

Friedman, J. 2003. Globalization, Dis-integration, Re-organisation. The Transformation of Violence'. In Globalization, the State, and Violence, hrsg J. Friedman, 1-34. Walnut Creek, Altamira Press.

Gingrich, A. und M. Banks (Hrsg.). 2006. Neo-nationalism in Western Europe and Beyond. Perspectives from Social Anthropology. Oxford, New York: Berghahn.

Glazer, N. 1997. We Are All Multiculturalists Now! Cambridge: Harvard University Press.

Glick-Schiller, N. 2009. A Global Perspective on Migration and Development. Social Analysis, 53 (3): 14-37.

Glick-Schiller, N. L.Basch und C. Szanton Blanc. 1995. From Immigrant to Transmigrant. Theorizing Transnational Migration. Anthropological Quarterly 68 (1): 48-63. 
Glick-Schiller, N. L.Basch und C. Szanton Blanc. 1992. Towards a Transnational Perspective on Migration. Race, Class, Ethnicity, and Nationalism Reconsidered. New York: New York Academy of Sciences.

Glick-Schiller N. und A. Cağlar 2011. Locating Migration. Rescaling Cities and Migrants. Ithaca, New York: Cornell University Press.

Grillo, R. 2003. 'Cultural Essentialism and Cultural Anxiety'. Anthropological Theory 3, (2): 157-173.

Hastrup, K. 2002. Anthropology's comparative consciousness: the case of human rights. In Anthropology by Comparison. A. Gingrich und R. Fox 27-43. London, New York.

Hess, S., J. Binder und J. Moser (Hrsg.). 2009. No integration?! Kulturwissenschaftliche Beiträge zur Integrationsdebatte. In Europa. Bielefeld: transcript.

Holzleithner, E. und S. Strasser. (2010). Multikulturalismus im Widerstreit. Debatten über kulturelle Diversität, Geschlechtergleichheit und sexuelle Autonomie. In Multikulturalismus queer gelesen. Zwangsheirat und gleichgeschlechtliche Ehe in pluralen Gesellschaften, hrsg Strasser, S. und E. Holzleithner 27-46. Frankfurt/Main: Campus.

Joppke, C. 2004. The retreat of multiculturalism in the liberal state: theory and politics. The British Journal of Sociology 55 (2): 235-275.

Joppke, C. 2007. 'Beyond national models: Civic integration policies for immigrants in Western Europe'. West European Politics 30 (1): 1-22.

King, D. 2005. Facing the future: America's post-multiculturalist trajectory. Social Policy and Administration 39 (2): 116-29.

Koopmans, R. 2008. Tradeoffs between Equality and Difference. Immigrant Integration, Multiculturalism, and the Welfare State in Cross-National Perspective. Working Paper Wissenschaftszentrum Berlin für Sozialforschung. http://www.wzb.eu/zkd/mit/pdf/dp_sp_iv_2008-701.pdf. (20 January 2010).

Koopmans, R. 2010. 'Tradeoffs between Equality and Difference. Immigrant Integration, Multiculturalism and the Welfare State in Cross-National Perspective. Journal of Ethnic and Migration Studies 36 (1): 1-26. 
Koopmanns, R. und P. Statham (Hrsg..). 2000. Challenging Immigration and Ethnic Relations Politics: Comparative European Perspectives. Oxford: Oxford University Press.

Koopmanns, R., P. Statham, M. Giuigni und F. Passy. 2005. Contested Citizenship. Immigration and Cultural Diversity in Europe. Minneapolis, London: University of Minnesota.

Kymlicka, W. 1995. Multicultural Citizenship. A Liberal Theory of Minority Right. Oxford: Clarendon Press.

Kymlicka, W. (1998). Introduction: An Emerging Consensus? Ethical Theory and Moral Practice 1, 143-157.

Kymlicka, W. 2007. Multicultural Odysseys. Navigating the New International Politics of Diversity. Oxford: Oxford University Press.

Kymlicka, W. 2010. The rise and the fall of multiculturalism? New debates on inclusion and accommodation in diverse societies. In The multiculturalism backlash. European discourses, policies and practices hrsg S. Vertovec und S. Wessendorf, 32-49. Oxon: New York, Routledge.

Modood, T. und P. Werbner, Pnina. (Hrsg.). 1997. The Politics of Multiculturalism in a New Europe. Racism, Identity, Community. London, New York: Zed Books.

Okin, S. M. 1999. Is Multiculturalism Bad for Women? Princeton, New Jersey: Princeton University Press.

Parekh, B. 2000. Rethinking Multiculturalism. Cultural Diversity and Political Theory. Cambridge (Massachusetts), London: Palgrave.

Perchinig, B. 2010. 'All you Need to Know to Become an Austrian'. In A Redefinition of Belonging. Language and Integration Tests in Europe hrsg R. van Oers, E. Ersbøll und D. Kostakopoulou 25-50. Leiden: Brill.

Phillips, A. 2010. Einwilligung, Autonomie und Zwang: Erfahrungen in Großbritannien. In Multikulturalismus queer gelesen. Zwangsheirat und gleichgeschlechtliche Ehe in pluralen Gesellschaften hrsg S. Strasser und E. Holzleithner 182-201. Frankfurt/Main: Campus. 
Phillips, A. 2007. Multiculturalism Without Culture. Princeton, New Jersey: Princeton University Press.

Phillips, A. und S. Saharso. 2008. Guest editorial: The rights of women and the crisis of multiculturalism. Ethnicities 8(3): 291-301.

Prins, P. und S. Saharso. 2010. From toleration to repression: the Dutch backlash against mutliculturalism. In: The multiculturalism backlash. European discourses, policies and practices hrsg S. Vertovec und S. Wessendorf 72-91. Oxon, New York: Routledge.

Rattansi, A. 2004. Dialogues on difference: cosmopolitans, locals and 'others' in a postnational age. Sociology 38: 613-21.

Razack, S. H. 2004. Imperilled Muslim Women, Dangerous Muslim Men and Civilized Europeans: Legal and Social Responses to Forced Marriages. Feminist Legal Studies 12 (2): 129-174.

Römhild, R. 2009. Aus der Perspektive der Migration. Die Kosmopolitisierung Europas. In No integration?! Kulturwissenschaftliche Beiträge zur Integrationsdebatte in Europa, hrsg Hess, S., J. Binder und J. Moser 225-238. Bielefeld: transcript.

Saharso, S. 2008. Gibt es einen multikulturellen Feminismus? Ansätze zwischen Universalismus und Anti-Essenzialismus. In Zwangsfreiheiten. Multikulturalität und Feminismus, hrsg B. Sauer und S. Strasser 11-27. Wien: Promedia und Südwind.

Scheffer, P. 2000. Het multikulturele drama. NRC Handelsblad, 29. Jänner 2000.

Schiffauer, W. 2008. Parallelgesellschaften. Wie viel Wertekonsens braucht unsere Gesellschaft? Für eine kluge Politik der Differenz. Bielefeld: transcript.

Schiller, M. 2010. Zwangsverheiratung im Fokus. Ein Vergleich von Auftragsstudien in europäischen Ländern. In Multikulturalismus queer gelesen. Zwangsheirat und gleichgeschlechtliche Ehe in pluralen Gesellschaften, hrsg Strasser, S. und E. Holzleithner 47-70. Frankfurt/Main: Campus.

Schönwälder, K. 2010. Integration policy and pluralism in a self-conscious country of immigration. In The multiculturalism backlash. European discourses, policies and practices, hrsg S. Vertovec und S. Wessendorf 152-169. Oxon, New York: Routledge. 
Shachar, A. 2000. On Citizenship and Multicultural Vulnerability. Political Theory 28 (1): 64-89.

Strasser, S. 2010. Ist der Multikulturalismus noch zu retten? Ein Konzept auf dem Prüfstein von Wissenschaft Politik und Recht. In Multikulturalismus queer gelesen. Zwangsheirat und gleichgeschlechtliche Ehe in pluralen Gesellschaften hrsg Strasser, S. und E. Holzleithner 342-366. Frankfurt/Main: Campus.

Strasser, S. 2012. 'Rethinking Citizenship: Critical Encounters with Feminist, Multicultural and Transnational Concepts of Citizenship'. In Remaking Citizenship in Multicultural Europe: Women's Movements, Gender and Diversit, hsrg. B. Halsaa, S. Roseneil und S. Sümer 21-40. Basingstoke: Palgrave Macmillan.

Strasser, S. und E. Holzleithner (Hrsg.). 2010. Multikulturalismus queer gelesen. Zwangsheirat und gleichgeschlechtliche Ehe in pluralen Gesellschaften. Frankfurt/Main: Campus.

Sezgin, H. (Hrsg.). 2011. Manifest der Vielen. Deutschland erfindet sich neu. Berlin: Blumenbar Verlag.

Taylor, C. 1993. Multikulturalismus und die Politik der Anerkennung. Frankfurt am Main: Suhrkamp.

Turner, T. 1993. Anthropology and Multiculturalism: What is Anthropology That Multiculturalists Should Be Mindful of It? Cultural Anthropology 8 (4): 411-429.

Uitermark, J., U. Rossi und H. Van Houtum. 2005. Reinventing Multiculturalism: Urban Citizenship and the Negotiation of Ethnic Diversity in Amsterdam. International Journal of Urban and Regional Research 29 (3): 622-640.

Vertovec, S. 2007. Super-diversity and its implications. Ethnic and Racial Studies 30 (6): 1024-1054.

Vertovec, S. 2010. Towards post-multiculturalism? Changing communities, conditions and contexts of diversity. International Social Science Journal 61(199): 83-95. 
Vertovec, S. und S. Wessendorf. 2010. Introduction: assessing the backlash against multiculturalism in Europe. In The multiculturalism backlash. European discourses, policies and practice, hrsg S. Vertovec und S. Wessendorf 1-31. Oxon, New York: Routledge.

Volpp, L. 2001. Feminism versus Multiculturalism. Columbia Law review 101(5): 1181-1218.

Wikan, U. 2002. Generous Betrayal. Politics of Culture in the New Europe. Chicago:

University of Chicago Press.

Wimmer A. und N. Glick-Schiller. 2002. Methodological Nationalism and Beyond. NationState Building, Migration and the Social Sciences. Global Networks 2 (4): 301-334.

Young, I. M. 1989. Polity and Group Difference: A Critique of the Ideal of Universal Citizenship. Ethics 99, 250-274.

Young, I. M. 2007. Structural injustice in the politics of difference. In Multiculturalism and Political Theory hrsg A. Laden und D. Owen Cambridge (UK).

Yuval-Davis, N. 2006. Intersectionality and Feminist Politics. European Journal of Women's Studies 13 (3): 193-209.

Žižek, S. 2008. Tolerance as an ideological category. Critical Inquiry 34, 660-682. 